\title{
Correction: Accuracy of the FMF Bayes theorem-based model for predicting preeclampsia at 11-13 weeks of gestation in a Japanese population
}

\author{
Minako Goto - Keiko Koide - Mayumi Tokunaka - Hiroko Takita - Shoko Hamada - Masamistu Nakamura • \\ Ryu Matsuoka · Akihiko Sekizawa · Liona C. Poon
}

Published online: 23 December 2020

(c) The Japanese Society of Hypertension 2020, corrected publication 2021

Correction to: Hypertension Research

$$
\text { https://doi.org/10.1038/s41440-020-00571-4 }
$$

The original version of the Supplementary Information associated with this article contained errors in Supplementary Tables 1, 2 and Table 2. The corrected figures are shown below.

In the version of this article originally published, GA at screening and CRL in Supplementary Table 1, 2 and Table 2 contained several errors. In Supplementary Table 1, GA at screening (days) in Preterm PE HR-group should be
91 (80-96), those in Preterm PE LR-group should be 91 (77-97). CRL (mm) in Preterm PE HR-group should be 69 (49-85), those in Preterm PE LR-group should be 70 (40-85). In Supplementary Table 2, GA at screening (days) in Term PE HR-group should be 91 (77-96), those in Term PE LR-group should be 91 (78-97). CRL (mm) in Term PE HR-group should be 70 (40-85), those in Term PE LRgroup should be 70 (44-85). In Table 2, GA at screening (days) in Unaffected group should be 91 (77-97).

The original article has been corrected.
Table 2 Comparisons of maternal characteristics and biomarkers between outcome groups

\begin{tabular}{lcccr}
\hline Variable & $\begin{array}{l}\text { Preterm PE } \\
(n=11)^{\mathrm{a}}\end{array}$ & $\begin{array}{l}\text { Term PE } \\
(n=15)^{\mathrm{a}}\end{array}$ & $\begin{array}{l}\text { Unaffected } \\
(n=887)^{\mathrm{a}}\end{array}$ & $p$ value \\
\hline Maternal age (years) & $40(34-47)$ & $35(27-40)$ & $34(22-45)$ & $<0.05$ \\
Maternal weight (kg) & $56(47-86)$ & $52(40-75)$ & $52(37-95)$ & 0.11 \\
Maternal height (cm) & $158(154-168)$ & $157(147-170)$ & $159(140-180)$ & 0.68 \\
BMI $\left(\mathrm{kg} / \mathrm{m}^{2}\right)$ & $21.7(19.8-30.5)$ & $20.5(17.3-30.8)$ & $20.4(13.7-36.2)$ & $<0.05$ \\
Primipara & $73 \%(8)$ & $73 \%(11)$ & $53 \%(469)$ & 0.12 \\
In vitro fertilization & $55 \%(6)$ & $40 \%(6)$ & $23 \%(201)$ & $<0.05$ \\
Smoking & $18 \%(2)$ & $0 \%(0)$ & $6 \%(49)$ & 0.37 \\
CH & $36 \%(4)$ & $13 \%(2)$ & $0.2 \%(2)$ & $<0.05$ \\
DM & $9 \%(1)$ & $0 \%(0)$ & $0.3 \%(3)$ & 0.10 \\
Family history of PE & $0 \%(0)$ & $7 \%(1)$ & $4 \%(34)$ & 0.57 \\
Parous with previous PE & $0 \%(0)$ & $91(8)$ & $0.6 \%(6)$ & 0.84 \\
GA at screening (days) & $92(85-96)$ & $1.3(0.6-2.6)$ & $1.8(0.5-3.5)$ & 0.854 \\
UtA-PI & $2.0(0.9-3.2)$ & $0.84(0.32-1.58)$ & $1.09(0.34-2.44)$ & 0.06 \\
UtA-PI MoM & $1.26(0.63-2.06)$ & $97(71-115)$ & $80(55-110)$ & $<0.13$ \\
MAP (mmHg) & $91(78-125)$ & $1.08(0.87-1.28)$ & $0.97(0.70-1.31)$ & $<0.05$ \\
MAP MoM & $1.09(0.96-1.28)$ & $38.7(15.5-134.8)$ & $36.5(5.1-124.2)$ & $<0.05$ \\
PIGF (pg/mL) & $19.1(1.1-63.6)$ & $0.82(0.31-2.19)$ & $0.72(0.09-2.38)$ & $<0.05$ \\
PIGF MoM & $0.43(0.02-0.98)$ &
\end{tabular}

$B M I$ body mass index, $C H$ chronic hypertension, $D M$ diabetes mellitus, GA gestational age, MAP mean arterial pressure, $P E$ preeclampsia, $P I G F$ maternal serum placental growth factor, UtA-PI uterine artery pulsatility index

${ }^{a}$ The results are expressed as either median (range) or \% (n) 